\title{
A contribuição da consulta de enfermagem frente aos fatores psicossociais relacionados à adesão ao tratamento de insulinoterapia
}

The contribution of the nursing consultation facing the psychosocial factors related to the adherence to insulin therapy treatment

La contribución de la consulta de enfermería frente a los factores psicosociales relacionados con la adherencia al tratamiento de insulinoterapia

\section{Resumo}

Introdução: A Diabetes Mellitus é um grupo de distúrbios metabólicos que tem por característica um desequilíbrio no nível sérico da glicemia. Considerada um dos principais problemas de saúde pública, devido a sua capacidade de evoluir para complicações crônicas e agudas, enfrenta uma inadequada adesão ao autocuidado, especialmente, a insulinoterapia. Portanto, o estudo teve por objetivo conhecer a contribuição da consulta de enfermagem frente aos fatores psicossociais na adesão ao tratamento da insulinoterapia. Metodologia: A pesquisa foi norteada pelo método da Revisão Integrativa, realizada na Biblioteca Virtual de Saúde (BVS), nas seguintes bases de dados com acesso on-line: Base de Dados de Enfermagem (BDENF), Literatura Latino Americana e do Caribe em Ciências da Saúde (LILACS) e MEDLINE. Assim como, no PubMed Central (PMC) e no SciElo Brasil, ambos através do Portal de Periódicos CAPES. Resultados e discussão: De acordo com a literatura levantada, o autocuidado foi considerado um fator de suma importância no sucesso do tratamento dos pacientes diabéticos em geral. Aspectos psicossociais estão relacionados à qualidade da adesão a insulinoterapia e a consulta de enfermagem é relevante no processo de construção de autoconhecimento e um fator de adesão ao autocuidado. Conclusão: A consulta de enfermagem é uma ferramenta eficaz na adesão adequada à insulinoterapia. No entanto, o planejamento feito em consonância com o paciente para atender suas necessidades, deve fortalecer aspectos de protagonismo do autocuidado e evitar situações de dependência da equipe de saúde desnecessárias, que afetariam a qualidade de vida do indivíduo.

Palavras-chave: Autocuidado; Diabetes; Enfermagem; Insulina.

\begin{abstract}
Introduction: Diabetes Mellitus is a group of metabolic disorders that has as a characteristic an imbalance in the level of serum glucose. It is considered a major public health problem, due to its ability to develop into chronic and acute complications, and faces inadequate adherence to self-care, especially insulin therapy. Therefore, this study aimed to understand the contribution of the nursing consultation regarding psychosocial factors in the adherence to insulin therapy. Methodology: The research was guided by the Integrative Review method, carried out in the Virtual Health Library (VHL), in the following databases with on-line access: Nursing Database (BDENF), Latin American and Caribbean Literature on Health Sciences (LILACS) and MEDLINE. As well as, in PubMed Central (PMC) and SciElo Brazil, both through the CAPES Periodical Portal. Results and discussion: According to the literature surveyed, selfcare was considered a factor of paramount importance in the success of the treatment of diabetic patients in general. Psychosocial aspects are related to the quality of adherence to insulin therapy and the nursing consultation is relevant in the process of building self-knowledge and a factor of adherence to self-care. Conclusion: The nursing consultation
\end{abstract}


is an effective tool for adequate adherence to insulin therapy. However, planning done in agreement with the patient to meet their needs, should strengthen aspects of self-care protagonism and avoid situations of unnecessary dependence on the health team, which would affect the quality of life of the individual.

Keywords: Self-care; Diabetes; Nursing; Insulin.

\section{Resumen}

Introducción: La diabetes mellitus es un grupo de trastornos metabólicos que tiene como característica un desequilibrio en el nivel de glucosa sérica. Se considera un importante problema de salud pública, debido a su capacidad para desarrollar complicaciones crónicas y agudas, y se enfrenta a una inadecuada adherencia al autocuidado, especialmente a la terapia con insulina. Por lo tanto, este estudio tenía como objetivo conocer la contribución de la consulta de enfermería respecto a los factores psicosociales en la adherencia a la terapia de insulina. Metodología: La investigación fue guiada por el método de Revisión Integrativa, realizada en la Biblioteca Virtual en Salud (BVS), en las siguientes bases de datos con acceso en línea: Base de Datos de Enfermería (BDENF), Literatura Latinoamericana y del Caribe en Ciencias de la Salud (LILACS) y MEDLINE. Así como, en PubMed Central (PMC) y SciElo Brasil, ambos a través del Portal de Publicaciones Periódicas de CAPES. Resultados y discusión: Según la bibliografía consultada, el autocuidado se considera un factor de suma importancia para el éxito del tratamiento de los pacientes diabéticos en general. Los aspectos psicosociales están relacionados con la calidad de la adhesión a la insulinoterapia y la consulta de enfermería es relevante en el proceso de construcción del autoconsciente y un factor de adhesión al autocuidado. Conclusión: La consulta de enfermería es un instrumento eficaz en la administración adecuada de la insulinoterapia. Sin embargo, la planificación realizada de acuerdo con el paciente para satisfacer sus necesidades debe reforzar los aspectos de protagonismo del autocuidado y evitar situaciones de dependencia innecesaria del equipo de salud, lo que afectaría a la calidad de vida del individuo.

Palabras clave: Autocuidado; Diabetes; Enfermería; Insulina.

\section{Introdução}

A Diabetes Mellitus (DM) é um grupo de distúrbios metabólicos que tem por característica um desequilíbrio no nível sérico da glicemia, mais comumente identificada como um quadro persistente de hiperglicemia associada a complicações, disfunções e insuficiência de vários órgãos (Brasil, \& Ministério da Saúde [MS], 2013). Considerada um dos principais problemas de saúde pública, não apenas no Brasil, como no mundo, devido a sua grande capacidade de desenvolvimento de complicações tanto agudas quanto crônicas (Barbosa \& Souza, 2018).

Somente em 2019, houve 136 mil internações por esta causa, o que custou ao Sistema Único de Saúde o montante de 98 milhões de reais, mais de 6 vezes os custos das internações por Hipertensão Arterial Sistêmica (HAS) no mesmo ano, ainda que a HAS atinja 3,7 mais brasileiros do que a DM. Ademais, no ano de 2018, foram registrados 65 mil óbitos por DM no país contra 25 mil por HAS (Brasil \& MS, 2020). Concomitantemente, numa perspectiva ampliada, estima-se que mundialmente cerca de 387 milhões de pessoas convivam com esse quadro atualmente e que alcance o patamar de 471 milhões em 2035 (Moreira et al., 2018)

Quanto à classificação proposta pela Associação Americana de Diabetes - ADA, existem quatro tipos principais. Naqueles casos onde, devido à destruição das células beta do pâncreas, ocorre uma deficiência completa de insulina, é feito o diagnóstico de Diabetes Mellitus Tipo 1 (DMT1), ou os chamados insulinodependentes. A DMT1 surge em geral na infância ou na adolescência e é de origem autoimune ou idiopática (Moreira et al., 2018).

Quando a hiperglicemia estiver associada a graus variados de diminuição de secreção e/ou resistência à insulina, classifica-se como Diabetes Mellitus tipo 2 (DMT2). Outros Tipos Específicos de Diabetes estão relacionados a causas variadas, tais como as infecções, endocrinopatias, defeitos genéticos da ação da insulina, entre outros, inclusive a indução da diabetes por drogas ou produtos químicos. E, por fim, a diabetes gestacional, alusiva diretamente ao período gravídico e desaparecendo comumente ao final da gestação (Brasil \& MS, 2013).

Para alguns destes pacientes, e especialmente para os portadores de DMT1, o tratamento inclui o uso de insulinas injetáveis, cujo uso é diário e as doses variam conforme prescrição médica (Brasil \& MS, 2013). No entanto, a inadequada autoadministração ou baixa adesão a este autocuidado estão associadas ao aumento da morbimortalidade destes. Portanto, o 
estudo teve por objetivo conhecer a contribuição da consulta de enfermagem frente aos fatores psicossociais na adesão ao tratamento da insulinoterapia. Tendo em vista que, esses fatores são fundamentais para o estímulo ao autocuidado, a adequada orientação e a autopercepção do insulinodependente de seu estado de saúde, propiciam maior segurança na autoadministração do medicamento e devem ser trabalhados pelo enfermeiro durante a consulta de enfermagem (Brasil, \& MS, 2013).

\section{Metodologia}

A pesquisa foi norteada pelo método da Revisão Integrativa, realizada na Biblioteca Virtual de Saúde (BVS), nas seguintes bases de dados com acesso on-line: Base de Dados de Enfermagem (BDENF), Literatura Latino Americana e do Caribe em Ciências da Saúde (LILACS) e MEDLINE. Assim como, no PubMed Central (PMC) e no SciElo Brasil, ambos através do Portal de Periódicos CAPES. A pesquisa ocorreu no período de agosto de 2020 a fevereiro de 2021 e envolveu artigos que se encaixaram aos descritores: "Autocuidado", "Diabetes", "Enfermagem” " "Insulina”. Outrossim, utilizou-se o operador booleano AND para os seguintes cruzamentos realizados de acordo com os descritores pesquisados nas bases de dados: "Autocuidado" $A N D$ "Insulina", "Enfermagem" AND "Autocuidado" AND "Insulina", "Nursing" AND "Selfcare" AND "Diabetes" AND "Insulin" e "Enfermagem" AND "Autocuidado" AND "Diabetes".

Os critérios de inclusão estabelecidos para a revisão foram artigos disponíveis na íntegra, completos, gratuitos, nos idiomas português e inglês, publicados dentro dos últimos cinco anos. Sendo excluídos aqueles relacionados a cuidados intrahospitalares, à terapia endovenosa de insulina e duplicados. Ademais, com vistas ao aprofundamento da temática, foram consultadas publicações oficiais do Ministério da Saúde do Brasil, o Caderno de Atenção Básica 36 e o livro Situação de Hipertensão e Diabetes no Brasil 2020, disponíveis no acervo online deste órgão. A pergunta norteadora utilizada para o desenvolvimento do trabalho foi: Qual a contribuição da consulta de enfermagem frente aos fatores psicossociais que limitam uma boa adesão ao autocuidado, em especial a insulinoterapia, nos portadores de diabetes mellitus?

Tabela 1 - Seleção e inclusão de artigos após o levantamento bibliográfico na literatura.

\begin{tabular}{|c|c|c|c|}
\hline Etapa & $\begin{array}{c}\text { Portal } \\
\text { consultado }\end{array}$ & Descritores e operadores booleanos & Resultados \\
\hline \multirow{4}{*}{$\begin{array}{c}\text { Investigação } \\
\text { Artigos identificados } \\
\text { em cada base de } \\
\text { dados seguindo } \\
\text { critérios de inclusão }\end{array}$} & \multirow{2}{*}{ BVS } & "Autocuidado" $A N D$ "Insulina" & LILACS e BDENF: 75 \\
\hline & & "Enfermagem" $A N D$ “Autocuidado" $A N D$ "Insulina" & MEDLINE: 61 \\
\hline & \multirow{2}{*}{ CAPES } & $\begin{array}{c}\text { "Nursing" AND "Selfcare" AND "Diabetes" AND } \\
\text { "Insulin" }\end{array}$ & PubMed Central: 8 \\
\hline & & "Enfermagem" $A N D$ "Autocuidado" AND "Diabetes" & SciELO Brazil: 21 \\
\hline $\begin{array}{c}\text { Triagem } \\
\text { Estudos selecionados } \\
\text { para leitura de título } \\
\text { e resumos }\end{array}$ & & & $\begin{array}{c}\text { LILACS e BDENF: } \mathbf{3 5} \\
\text { MEDLINE: } \mathbf{7} \\
\text { PubMed Central: } \mathbf{2} \\
\text { SciELO Brazil: } \mathbf{1 4}\end{array}$ \\
\hline $\begin{array}{c}\text { Elegibilidade } \\
\text { Artigos não elegíveis } \\
\text { seguindo critérios de } \\
\text { exclusão }\end{array}$ & & & $\begin{array}{c}\text { Duplicados: } \mathbf{3} \\
\text { Fuga ao tema: } \mathbf{3 7}\end{array}$ \\
\hline Amostra Final & & & 18 \\
\hline
\end{tabular}

Fonte: Autores com resultados do estudo (2021). 
Após a leitura dos artigos na íntegra, foram incluídos aqueles que cumpriram um ou mais dos critérios estabelecidos a seguir: abordar aspectos psicossociais positivos e negativos, aderência ao autocuidado, adesão à insulinoterapia e contribuição da consulta de enfermagem. Destarte, 18 artigos elegíveis compuseram a amostra final.

\section{Resultados e Discussão}

Os artigos eleitos foram organizados no Quadro 1, de forma a permitir a análise dos resultados encontrados, caracterizados por título, autores, ano de publicação, tipo de estudo e resultados.

Quadro 1 - Caracterização dos artigos elegíveis segundo título, autores, ano de publicação, tipo de estudo e resultado.

\begin{tabular}{|c|c|c|c|c|}
\hline Título & Autores & $\begin{array}{c}\text { Ano de } \\
\text { publicação }\end{array}$ & Tipo de estudo & Resultado \\
\hline $\begin{array}{l}\text { Perspectivas para a } \\
\text { enfermagem face aos } \\
\text { avanços tecnológicos } \\
\text { no controle do diabetes. }\end{array}$ & $\begin{array}{l}\text { Barbosa, J.A.G., \& } \\
\text { Souza, M. C. M. R. }\end{array}$ & 2018 & $\begin{array}{l}\text { Estudo } \\
\text { descritivo com } \\
\text { base em uma } \\
\text { revisão de } \\
\text { literatura }\end{array}$ & $\begin{array}{l}\text { O mercado oferece diferentes recursos para o } \\
\text { tratamento da doença perpassando por insulinas de } \\
\text { ação mais acurada, com formas de administração } \\
\text { mais fáceis, menos dolorosas e mais seguras, como } \\
\text { as canetas injetoras. Já se dispõe da possibilidade de } \\
\text { monitoramento das taxas de glicemia sem a } \\
\text { necessidade de picadas frequentes de dedo e, ainda, } \\
\text { da infusão contínua de insulina também sem a } \\
\text { necessidade de injeções múltiplas no subcutâneo } \\
\text { visando uma melhor adesão ao tratamento e controle } \\
\text { glicêmico. Essas inovações se mostram, assim, } \\
\text { favorecedoras da prevenção de complicações, não só } \\
\text { aquelas de longo prazo, tão temidas com o avançar } \\
\text { do tempo da doença, mas, também, aquelas agudas, } \\
\text { como a hipoglicemia, que pode levar à morte. }\end{array}$ \\
\hline $\begin{array}{l}\text { Fatores relacionados à } \\
\text { autoaplicação de } \\
\text { insulina em indivíduos } \\
\text { com diabetes mellitus. }\end{array}$ & $\begin{array}{l}\text { Moreira, T. R., } \\
\text { Toledo, L. V., } \\
\text { Colodette, R. M., } \\
\text { Mendonça, É. T. de, } \\
\text { Amaro, M. de O. F., } \\
\text { Ayres, L. F. A., \& } \\
\text { Henriques, B. D. }\end{array}$ & 2018 & $\begin{array}{l}\text { Estudo } \\
\text { transversal }\end{array}$ & $\begin{array}{l}\text { A prevalência de autoaplicação de insulina foi de } \\
67,6 \% \text {, apresentando associação com idade entre } 57 \\
\text { e } 68 \text { anos (OR = } 0,3 ; \text { IC95\%: } 0,1-0,9) \text {, conviver com } \\
\text { companheiro e filhos (OR = 2,5; IC95\%: } 1,1-5,0) \text {, } \\
\text { ter } 9 \text { anos ou mais de estudo (OR = 8,4; IC95\%: } 1,9 \\
\text { - 37,9), morar em área não coberta pela Estratégia } \\
\text { Saúde da Família (ESF) (OR }=2,8 ; \text { IC95\%: } 1,1-7,0) \text {. }\end{array}$ \\
\hline $\begin{array}{l}\text { Comparative } \\
\text { effectiveness of a } \\
\text { technology-facilitated } \\
\text { depression care } \\
\text { management model in } \\
\text { safety-net primary care } \\
\text { Patients with type } 2 \\
\text { diabetes: } 6 \text {-month } \\
\text { outcomes of a large } \\
\text { clinical trial. }\end{array}$ & $\begin{array}{l}\text { Wu, S., Ell, K., Jin, } \\
\text { H., Vidyanti, I., } \\
\text { Chou, C.-P., Lee, P.- } \\
\text { J., Gross-Schulman, } \\
\text { S., Sklaroff, L. M., } \\
\text { Belson, D., Nezu, A. } \\
\text { M., Hay, J., Wang, } \\
\text { C.-J., Scheib, G., } \\
\text { Capua, P. D., } \\
\text { Hawkins, C., Liu, } \\
\text { P., Ramirez, M., } \\
\text { Wu, B. W., } \\
\text { Richman, M., } \\
\text { Myers, C., } \\
\text { Agustines, D., } \\
\text { Dasher, R., } \\
\text { Kopelowicz, A., } \\
\text { Allevato, J., Roybal, } \\
\text { M., Ipp, E., Haider, } \\
\text { U., Graham, S., } \\
\text { Mahabadi, V., \& } \\
\text { and Guterman, J. }\end{array}$ & 2018 & $\begin{array}{l}\text { Estudo } \\
\text { transversal }\end{array}$ & $\begin{array}{l}\text { Em comparação com os cuidados habituais, os } \\
\text { grupos de cuidados assistidos e de cuidados } \\
\text { facilitados por tecnologia foram associados a uma } \\
\text { redução significativa nos sintomas depressivos } \\
\text { medidos por pontuações no Questionário de Saúde } \\
\text { do Paciente. }\end{array}$ \\
\hline
\end{tabular}




\begin{tabular}{|c|c|c|c|c|}
\hline $\begin{array}{l}\text { Adesão aos cuidados } \\
\text { com o diabetes mellitus } \\
\text { em três níveis de } \\
\text { atenção à saúde. }\end{array}$ & $\begin{array}{l}\text { Souza, J. D., } \\
\text { Baptista, M. H. B., } \\
\text { Gomides, D. S., \& } \\
\text { Pace, A. E. }\end{array}$ & 2017 & $\begin{array}{l}\text { Estudo } \\
\text { transversal } \\
\text { quantitativo e } \\
\text { descritivo }\end{array}$ & $\begin{array}{l}\text { A adesão para a atividade física, monitorização da } \\
\text { glicemia capilar, inspeção dos calçados e uso de } \\
\text { insulina diferiu entre as unidades, com melhores } \\
\text { resultados na atenção terciária. }\end{array}$ \\
\hline $\begin{array}{l}\text { The role of } \\
\text { psychological insulin } \\
\text { resistance in diabetes } \\
\text { self-care management. }\end{array}$ & Lim, A., \& Song, Y. & 2020 & $\begin{array}{l}\text { Estudo } \\
\text { transversal } \\
\text { descritivo }\end{array}$ & $\begin{array}{l}\text { A autoeficácia do diabetes }(\mathrm{r}=.53, \mathrm{p}<.001) \text { e a } \\
\text { resistência psicológica à insulina }(\mathrm{r}=.33, \mathrm{p}<.001) \\
\text { foram significativamente associadas ao autocuidado } \\
\text { do diabetes, enquanto uma associação negativa foi } \\
\text { encontrada entre a eficiência do autocuidado e a } \\
\text { resistência psicológica à insulina ( } \mathrm{r}=-, 16 \text {, p } \\
<0,001) \text {. Quando a resistência psicológica à insulina } \\
\text { foi inserida como um mediador, a associação entre a } \\
\text { autoeficácia e o gerenciamento do autocuidado do } \\
\text { diabetes foi atenuada. }\end{array}$ \\
\hline $\begin{array}{l}\text { Barriers of adherence } \\
\text { and possible solutions } \\
\text { to nonadherence to } \\
\text { antidiabetic therapy in } \\
\text { women with diabetes in } \\
\text { pregnancy: patients, } \\
\text { perspective }\end{array}$ & $\begin{array}{l}\text { Mukona, D., } \\
\text { Munjanja, S. P., } \\
\text { Zvinavashe, M., \& } \\
\text { Stray-Pederson, B. }\end{array}$ & 2017 & $\begin{array}{l}\text { Estudo } \\
\text { qualitativo } \\
\text { descritivo }\end{array}$ & $\begin{array}{l}\text { As barreiras identificadas foram o baixo nível } \\
\text { socioeconômico, a falta de apoio familiar, de pares e } \\
\text { da comunidade, efeitos da gravidez, regime } \\
\text { terapêutico complicado, fisiopatologia do diabetes, } \\
\text { crenças culturais e religiosas e sistema de saúde } \\
\text { precário. } \\
\text { As soluções possíveis foram fomentar o apoio social, } \\
\text { o apoio financeiro e a melhoria dos serviços } \\
\text { hospitalares. O cuidado individualizado de mulheres } \\
\text { com diabetes é essencial, e as barreiras e possíveis } \\
\text { soluções identificadas podem ser utilizadas para } \\
\text { melhorar o cuidado. }\end{array}$ \\
\hline $\begin{array}{l}\text { Comparação das } \\
\text { estratégias educativas } \\
\text { em diabetes mellitus: } \\
\text { educação em grupo e } \\
\text { visita domiciliar }\end{array}$ & Santos, J. C. & 2018 & $\begin{array}{l}\text { Ensaio clínico } \\
\text { com cluster } \\
\text { randomizado }\end{array}$ & $\begin{array}{l}\text { Observou-se melhora nos aspectos relacionados ao } \\
\text { empoderamento e adesão ao autocuidado tanto da } \\
\text { educação em grupo quanto na visita domiciliar. } \\
\text { Entretanto, apenas na educação em grupo houve } \\
\text { melhora estatisticamente significativa em relação à } \\
\text { hemoglobina glicada. }\end{array}$ \\
\hline $\begin{array}{l}\text { Efeito da consulta de } \\
\text { enfermagem no } \\
\text { conhecimento, } \\
\text { qualidade de vida, } \\
\text { atitude frente à doença } \\
\text { e autocuidado em } \\
\text { pessoas com diabetes }\end{array}$ & $\begin{array}{l}\text { Teston, E. F., } \\
\text { Peternella, F. M. N., } \\
\text { Sales, C. A., } \\
\text { Haddad, M. C. L., } \\
\text { Cubas, M. R., \& } \\
\text { Marcon, S. S. }\end{array}$ & 2018 & $\begin{array}{l}\text { Ensaio clínico } \\
\text { randomizado e } \\
\text { controlado }\end{array}$ & $\begin{array}{l}\text { A consulta de enfermagem promoveu mudança } \\
\text { significativa em relação ao conhecimento sobre o } \\
\text { diabetes }(p<0,001) \text {, impacto da doença na qualidade } \\
\text { de vida }(p=0,002) \text {, na atitude frente à doença } \\
(p=0,024) \text { e na adesão às atividades de autocuidado } \\
(p<0,001) \text {. }\end{array}$ \\
\hline $\begin{array}{l}\text { Autocuidado em } \\
\text { pessoas com diabetes } \\
\text { mellitus tipo 1: } \\
\text { vivências de } \\
\text { adolescentes }\end{array}$ & $\begin{array}{l}\text { Fragoso, L. V. C., } \\
\text { Cunha, M. C. S. O., } \\
\text { Fragoso, E. B., \& } \\
\text { Araújo, M. F.M. }\end{array}$ & 2019 & $\begin{array}{l}\text { Estudo } \\
\text { qualitativo }\end{array}$ & $\begin{array}{l}\text { Com base nos dados desvelados observou-se os } \\
\text { seguintes enfrentamentos na adesão ao autocuidado; } \\
\text { dilemas de ser adolescente com diabetes e ações do } \\
\text { profissional de saúde e da família para o autocuidado } \\
\text { do adolescente. }\end{array}$ \\
\hline $\begin{array}{l}\text { Competência para o } \\
\text { autocuidado na } \\
\text { administração de } \\
\text { insulina por } \\
\text { septuagenários ou mais } \\
\text { idosos. }\end{array}$ & Vianna, M. S. & 2016 & $\begin{array}{l}\text { Estudo } \\
\text { transversal }\end{array}$ & $\begin{array}{l}\text { Na avaliação dos erros na autoaplicação de insulina, } \\
\text { a maior frequência foi na lavagem das mãos }(87,2 \%) \text {, } \\
\text { na aspiração de ar na seringa e injeção no frasco de } \\
\text { insulina }(74,3 \%) \text { e no descarte de material } \\
\text { perfurocortante em frasco rígido }(73,1 \%) \text {. }\end{array}$ \\
\hline $\begin{array}{l}\text { Differences in foot self- } \\
\text { care and lifestyle } \\
\text { between men and } \\
\text { women with diabetes } \\
\text { mellitus }\end{array}$ & $\begin{array}{l}\text { Rossaneis, M. A., } \\
\text { Haddad, M. C. F. L., } \\
\text { Mathias, T. A. de F., } \\
\text { \& Marcon, S. S. }\end{array}$ & 2016 & $\begin{array}{l}\text { Estudo } \\
\text { transversal }\end{array}$ & $\begin{array}{l}\text { A prevalência de déficit de autocuidado com os pés } \\
\text { foi significativamente maior entre os homens, assim } \\
\text { como, também apresentaram comportamentos menos } \\
\text { saudáveis pois tem menor controle alimentar e não } \\
\text { realizam os exames laboratoriais referentes ao perfil } \\
\text { lipídico na frequência recomendada. }\end{array}$ \\
\hline $\begin{array}{l}\text { Evaluation of the } \\
\text { telephone intervention } \\
\text { in the promotion of } \\
\text { diabetes self-care: a } \\
\text { randomized clinical } \\
\text { trial }\end{array}$ & $\begin{array}{l}\text { Fernandes, B. S. M., } \\
\text { Reis, I. A., \& } \\
\text { Torres, H.C. }\end{array}$ & 2016 & $\begin{array}{l}\text { Ensaio clínico } \\
\text { randomizado }\end{array}$ & $\begin{array}{l}\text { O efeito benéfico no rendimento médio dos escores } \\
\text { de autocuidado no grupo experimental foi de } 1,03 \text { a } \\
1,78 \text { maior do que no grupo controle, com melhora } \\
\text { progressiva e significativa }(\mathrm{p}<0,001) \text {. }\end{array}$ \\
\hline
\end{tabular}




\begin{tabular}{|c|c|c|c|c|}
\hline $\begin{array}{l}\text { Relationship between } \\
\text { the users' contact time } \\
\text { in educational } \\
\text { programs on diabetes } \\
\text { mellitus and self-care } \\
\text { skills and knowledge }\end{array}$ & $\begin{array}{l}\text { Maia, M. A., Reis, } \\
\text { I.A., \& Torres, H.C. }\end{array}$ & 2016 & $\begin{array}{l}\text { Estudo } \\
\text { longitudinal } \\
\text { com abordagem } \\
\text { quantitativa }\end{array}$ & $\begin{array}{l}\text { A análise mostrou que a melhora nos escores de } \\
\text { autocuidado foi maior após uma intervenção } \\
\text { educacional de oito horas ou mais ( } \mathrm{p} \text {-valor }<0,05 \text { ). Os } \\
\text { escores de conhecimento teve melhora significativa } \\
\text { ao final do programa educacional. }\end{array}$ \\
\hline $\begin{array}{l}\text { Autocuidado e } \\
\text { percepção do } \\
\text { tratamento para o } \\
\text { diabetes por pessoas em } \\
\text { uso de insulina }\end{array}$ & $\begin{array}{l}\text { Reis, P. dos, Arruda, } \\
\text { G. O. de, Nass, E. } \\
\text { M. A., Ratuchnei, E. } \\
\text { S., Haddad, M. do } \\
\text { C. F. L., \& Marcon, } \\
\text { S. S. }\end{array}$ & 2020 & $\begin{array}{l}\text { Pesquisa } \\
\text { descritiva } \\
\text { exploratória de } \\
\text { natureza } \\
\text { qualitativa }\end{array}$ & $\begin{array}{l}\text { Emergiram três categorias que abordam as } \\
\text { dificuldades em mudar hábitos de vida, as } \\
\text { repercussões do uso de insulina na vida cotidiana e } \\
\text { os entraves vivenciados na aquisição dos insumos } \\
\text { farmacêuticos para o tratamento. }\end{array}$ \\
\hline $\begin{array}{l}\text { Intervenção educativa } \\
\text { em homens com } \\
\text { diabetes mellitus: } \\
\text { efeitos sobre } \\
\text { comportamentos e } \\
\text { perfil antropométrico }\end{array}$ & $\begin{array}{l}\text { Arruda, G. O. de, } \\
\text { Marcon, S. S., } \\
\text { Peruzzo, H. E., } \\
\text { Ruiz, A. G. B., } \\
\text { Back, I. R., Nass, E. } \\
\text { M.A., Batista, V. C., } \\
\text { \& Lino, I. G. T. }\end{array}$ & 2020 & $\begin{array}{l}\text { Ensaio clínico } \\
\text { randomizado } \\
\text { por clusters }\end{array}$ & $\begin{array}{l}\text { Observou-se o aumento da frequência semanal do } \\
\text { consumo de cereais e derivados ( } \mathrm{p}=0,033) \text {, carnes e } \\
\text { embutidos }(\mathrm{p}=0,003) \text {, diminuição do consumo de } \\
\text { raízes e tubérculos ( } \mathrm{p}=0,044) \text {. Constatou-se } \\
\text { diminuição discreta e não significativa da } \\
\text { circunferência da cintura, circunferência do quadril e } \\
\text { aa relação cintura-quadril, além do aumento não } \\
\text { significativo do peso, porcentagem de gordura } \\
\text { corporal e do índice de massa corporal. }\end{array}$ \\
\hline $\begin{array}{l}\text { Desempenho de } \\
\text { pessoas com diabetes } \\
\text { mellitus na } \\
\text { insulinoterapia }\end{array}$ & $\begin{array}{l}\text { Reis, P. dos; } \\
\text { Marcon, S. S.; Nass, } \\
\text { E. M. A.; Arruda, G. } \\
\text { O. de; Back, I. R.; } \\
\text { Lino, I. G. T.; } \\
\text { Francisqueti, V. }\end{array}$ & 2020 & $\begin{array}{l}\text { Estudo } \\
\text { transversal } \\
\text { descritivo }\end{array}$ & $\begin{array}{l}\text { Foram avaliados } 168 \text { usuários com idade média de } \\
69,9 \text { anos. O número de erros referentes ao processo } \\
\text { de aplicação da insulina variou de } 3 \text { a } 22 \text { de um total } \\
\text { de } 31 \text { questões. Verificou-se que } 96 \quad(56 \%) \\
\text { participantes faziam o descarte de material em local } \\
\text { inadequado e } 157 \quad(93,4 \%) \text { reutilizavam } \\
\text { seringas/agulhas. }\end{array}$ \\
\hline $\begin{array}{l}\text { Intervenção educativa } \\
\text { sobre o conhecimento e } \\
\text { manejo de insulina no } \\
\text { domicílio }\end{array}$ & $\begin{array}{l}\text { Reis, P. dos; } \\
\text { Marcon, S. S.; } \\
\text { Teston, E. F.; Nass, } \\
\text { E. M. A.; Ruiz, A. } \\
\text { G. B.; Francisqueti, } \\
\text { V.; Silva, E. S.; } \\
\text { Otero, L. M. }\end{array}$ & 2020 & $\begin{array}{l}\text { Estudo } \\
\text { quantitativo, do } \\
\text { tipo antes e } \\
\text { depois. }\end{array}$ & $\begin{array}{l}\text { Houve diferença nas frequências de acertos sobre o } \\
\text { conhecimento e administração de insulina antes e } \\
\text { após intervenção e, de } 31 \text { questões passíveis de } \\
\text { intervenção, esta se mostrou eficiente em } 96,77 \% \text {, e } \\
\text { em } 80,64 \% \text { foi estatisticamente significativa. }\end{array}$ \\
\hline $\begin{array}{l}\text { A percepção dos } \\
\text { usuários com diabetes } \\
\text { sobre a estratégia de } \\
\text { educação em grupos na } \\
\text { promoção do } \\
\text { autocuidado }\end{array}$ & $\begin{array}{l}\text { Vieira, G. de L. C., } \\
\text { Cecílio, S. G., } \\
\text { Torres, H. de C. }\end{array}$ & 2017 & $\begin{array}{l}\text { Estudo } \\
\text { descritivo de } \\
\text { abordagem } \\
\text { qualitativa }\end{array}$ & $\begin{array}{l}\text { As categorias evidenciadas pelo estudo } \\
\text { (aprendizagem por meio da participação nos grupos; } \\
\text { autocuidado em diabetes; superação de barreiras na } \\
\text { busca de um estilo de vida saudável; abordagem dos } \\
\text { sentimentos por meio da educação em grupos; e } \\
\text { benefícios relacionados à participação nos grupos) } \\
\text { demonstram que a estratégia de educação em grupo } \\
\text { contribui para a aprendizagem e estimula as práticas } \\
\text { de autocuidado em diabetes. }\end{array}$ \\
\hline
\end{tabular}

Fonte: Autores com resultados do estudo (2021).

A insulinoterapia é um importante mecanismo para a manutenção do nível glicêmico e qualidade de vida de portadores de diabetes (Souza, Baptista, Gomides, \& Pace, 2017). Todavia, a eficácia desse mecanismo varia de acordo com a qualidade da adesão ao mesmo, tendo em vista que resulta na diminuição considerável de complicações crônicas ou agudas relacionadas a este quadro, ou seja, aspectos psicossociais, culturais e econômicos influenciam diretamente à qualidade da adesão (Lim, \& Song, 2020).

Pessoas com contato precoce à insulinoterapia demonstram uma menor resistência psicológica à insulina. Conquanto, essa resistência é resultado não apenas de fatores intrínsecos, como falta de confiança do paciente para exercer o autocuidado e falta de conhecimento sobre a insulinoterapia, mas também de fatores externos, como deficiência do suporte familiar e social (Lim \& Song, 2020). Dessarte, foi possível encontrar padrões favoráveis, tanto clínicos, com redução percentual na hemoglobina glicada (HbA1c) (Santos, 2018), quanto comportamentais naqueles grupos submetidos a intervenções, que incluem 
acompanhamento ativo e consulta de enfermagem com ou sem visitas domiciliares, se comparados àqueles sujeitos apenas à orientação casual durante consulta de rotina (Souza, et al., 2017).

Em 2018, Teston et al realizou um estudo para qualificar o efeito da consulta de enfermagem (CE) sobre um grupo de portadores de DM. A maioria desses indivíduos inicialmente apresentavam conhecimento insatisfatório sobre a doença e atitude negativa frente a mesma. E, ainda que, parte desses indivíduos apresentasse baixo nível de sofrimento emocional, mostraram também baixa adesão ao autocuidado. Para estudar o efeito que essa intervenção norteada pelo autocuidado teria sobre esses indivíduos, foi feito o acréscimo de três CE ao Grupo de Intervenção (GI) e comparado os resultados com o Grupo de Controle (GC). Sendo então, verificado que, após a intervenção, houve uma significativa melhora em todos os aspectos outrora investigados no GI. Enquanto em GC, não ocorreu alteração relevante.

Outrossim, o desconforto relatado na administração da insulina também resulta em falhas na adesão à insulinoterapia, sobretudo em pacientes mais jovens ou familiares (normalmente pais), ou aqueles que estão em processo de adaptação à autoadministração, o ato de se "ferir" no momento da administração acarreta uma dor tanto física quanto psicológica. Decerto, atenuar o incômodo, é possível através do fortalecimento do apoio familiar. Cabendo aos profissionais de saúde envolvidos no processo de educação em saúde, promover melhora do conhecimento sobre a DM e autopercepção de sinais e sintomas inerentes aos agravos desta, de forma que resulta em maior sensação de segurança e por conseguinte, adesões mais sólidas ao tratamento (Fragoso, Cunha, Fragoso \& Araújo, 2019).

Ainda sobre esse aspecto, os idosos necessitam de atenção especial da equipe de Atenção Primária à Saúde (APS) e o enfermeiro deve avaliar a competência do idoso para este autocuidado. A predominância dos erros nessa faixa etária está relacionada à inadequada lavagem das mãos, na aspiração de ar na seringa e injeção no frasco de insulina e no descarte de material perfurocortante em frasco rígido (Vianna, 2016).

Para Vianna (2016), deve-se considerar que alguns pacientes apresentam maior probabilidade de realizar a autoaplicação de insulina. São eles os mais novos, com maior escolaridade e que moram com companheiros e filhos. Conforme ocorre o envelhecimento, atitudes negativas frente à doença ou diminuição do conhecimento e treinamento para a administração, a adesão à insulinoterapia sofre decréscimo.

Ademais, outro fator a ser considerado na qualidade do autocuidado é o gênero, visto que se identificaram disparidades sexuais significativas em uma análise transversal do Pathways Study. Comumente, as mulheres apresentaram piores resultados relacionados ao controle glicêmico e lipídico enquanto os homens demonstraram condutas mais inadequadas referentes aos cuidados com os pés (Rossaneis, Haddad, Mathias \& Marcon, 2016).

$\mathrm{Na}$ esfera de gerenciamento do autocuidado, a educação fornecida nos vários níveis de atenção à saúde é de grande valia para capacitar adequadamente o indivíduo. Logrando esse resultado através da formação de grupos de apoio e treinamento, onde os portadores de diabetes possam compartilhar vivências e adquirir técnica adequada aos diversos autocuidados (Santos, 2018). Contudo, nos casos onde não existe um direcionamento à autonomia do indivíduo, é possível que a consequência seja uma acomodação do paciente para a assistência prestada pela equipe de Atenção Primária à Saúde (APS) ou Estratégia de Saúde da Família (ESF) e resultar numa menor independência na autoadministração da insulina nessa população diabética adscrita (Moreira et al., 2018).

Em contrapartida, um ensaio clínico, realizado em Belo Horizonte, que visava avaliar a efetividade da intervenção telefônica na promoção do autocuidado, concluiu que esta tem efeito benéfico quando comparado ao acompanhamento convencional. Uma vez que o efeito médio dos escores do questionário de autocuidado aplicado antes, durante e após a intervenção no grupo-experimental obteve uma pontuação maior do que o grupo controle. Além de representar um novo campo de atuação para a educação em saúde (Fernandes, Reis, \& Torres, 2016). 
Um fator importante a ser observado, durante a intervenção telefônica, é a repetição das informações do processo de autocuidado tendo em vista que a mudança comportamental é uma ação continuada e gradativa. Uma constante exposição desses conhecimentos provoca reflexão sobre as atitudes de enfrentamento no próprio indivíduo, resultando numa maior vigilância sobre suas ações (Maia, Reis \& Torres, 2016).

Esse tipo de intervenção apresentou resultados satisfatórios, também, no rastreamento de sinais e sintomas de depressão no portador de diabetes. Este é um grupo com até o dobro de risco de desenvolver um quadro depressivo (Wu et al., 2018), portanto, uma consulta de enfermagem com busca ativa aliada a modelos de atenção em cuidado mental permite uma comunicação colaborativa e preventiva, inclusive, em quadros onde o paciente se sinta impotente no enfrentamento da diabetes (Lim \& Song, 2020). Dessa forma, quando comparado ao grupo de controle, pacientes que receberam ligações da equipe de apoio de enfermagem ou mesmo ligações automatizadas direcionadas a rastreamento desses sintomas, apresentaram significativa redução dos sinais depressivos em até seis meses, resultando em melhora da qualidade de vida, no controle glicêmico e na adesão aos diversos autocuidados, inclusive a insulinoterapia (Wu et al., 2018).

Em suma, o autocuidado é imprescindível para a manutenção da qualidade de vida do portador de DM, sobretudo, a adesão satisfatória ao tratamento com insulina. Nesse processo de cuidado do insulinodependente, ao enfermeiro e à equipe multiprofissional, cabe um papel de promover a educação e o treinamento essencial para uma administração segura e eficaz da insulina, preconizando o empoderamento e independência do indivíduo no que concerne ao autocuidado (Santos, 2018).

Além disso, é possível recorrer a estratégias educativas e apresentar alternativas tecnológicas com intuito de minimizar o desconforto físico e psicológico na autoaplicação do fármaco. Sendo assim, as intervenções de enfermagem devem ser planejadas de forma individual, observando aspectos sociais, culturais, econômicos e a faixa etária do paciente envolvido, caso contrário, pode resultar em frustrações e baixa adesão, sobretudo em idosos e gestantes (Mukona, Munjanja, Zvinavashe, \& Stray-Pederson, 2017; Vianna, 2016).

Dessa forma, a consulta de enfermagem que está em consonância com o cliente para atender suas necessidades é uma ferramenta eficaz na adesão adequada à insulinoterapia. Fortalecendo aspectos de protagonismo no autocuidado e evitando situações desnecessárias de dependência da equipe de saúde, que afetariam a qualidade de vida do portador de DM. Cabe ao enfermeiro estimular na família e no círculo social do paciente o apoio necessário para as boas práticas, não apenas referente à autoadministração de insulina, mas aos demais autocuidados como adesão à dieta e prática de atividades prescritas (Fragoso, Cunha, Fragoso, \& Araújo, 2019).

Ainda que um tema de relevância, levando-se em consideração a heterogenia da população brasileira, os gastos com internações e os óbitos precoces por esta causa, existe pouca pesquisa voltada para investigar a baixa adesão às consultas ou a adequada administração da insulina em diferentes regiões de saúde no território nacional. Decerto, conhecer esses dados seria de grande importância para o planejamento local da assistência desses pacientes com redução da evasão das consultas e tratamento, com repercussão direta na qualidade de vida dos pacientes e reduzindo despesas médicas com a assistência à saúde.

\section{Conclusão}

A consulta de enfermagem ocupa uma posição importante no que concerne à adesão do indivíduo ao autocuidado, seja a insulinoterapia ou outro, fornecendo apoio, orientações e identificando precocemente agravamentos de natureza psicológica e psiquiátrica, como a depressão. Essa adesão representa melhoras clínicas e de enfrentamento ao quadro de diabetes e resulta numa melhor qualidade de vida do portador de DM. Por conseguinte, para que a consulta atinja o objetivo proposto, é necessário considerar os motivos que resultam na baixa adesão, que varia de pessoa a pessoa, e planejar um autocuidado fornecendo uma capacitação voltada à independência desse cliente. 
Além disso, o acompanhamento por telefone desses indivíduos é uma opção com semelhante eficácia se comparada aos métodos presenciais de consulta, em casa ou na unidade de saúde, e deve ser considerado na busca ativa por sinais de falha no enfrentamento da doença e acompanhamento da evolução do quadro. Por certo, o objetivo do estudo foi alcançado e a pergunta norteadora respondida, uma vez que foi identificado a contribuição da consulta de enfermagem frente aos fatores psicossociais que limitam uma boa adesão ao autocuidado, em especial a insulinoterapia, nos portadores de diabetes mellitus. No entanto, se faz necessário o estímulo às pesquisas voltadas para melhor identificar esses fatores atenuantes e adjuvantes no público alvo e por localidade, de forma a servir de base para um melhor planejamento da assistência de enfermagem.

\section{Referências}

Arruda, G. O. de, Marcon, S. S., Peruzzo, H. E., Ruiz, A. G. B., Back, I. R., Nass, E. M.A., Batista, V. C., \& Lino, I. G. T. (2020). Intervenção educativa em homens com diabetes mellitus: efeitos sobre comportamentos e perfil antropométrico. Acta Paul. Enferm. (Online),33: eAPE20190128. http://www.revenf.bvs.br/scielo.php?script=sci_arttext\&pid=S0103-21002020000100423.

Barbosa, J.A.G., \& Souza, M.C.M.R. (2018). Perspectivas para a enfermagem face aos avanços tecnológicos no controle do diabetes. Rev. Enferm. UFPE on line, 12(4), 1112-1116. https://periodicos.ufpe.br/revistas/revistaenfermagem/article/view/231124/28692.

Brasil, \& Ministério da Saúde (2013). Caderno de Atenção Básica 36: Estratégias para o cuidado da pessoa com doença crônica: Diabetes Mellitus. Brasília: Ministério da Saúde. https://bvsms.saude.gov.br/bvs/publicacoes/estrategias_cuidado_pessoa_diabetes_mellitus_cab36.pdf.

Brasil, \& Ministério da Saúde (2020). Situação de Hipertensão e Diabetes no Brasil. Brasília: Ministério da Saúde. http://189.28.128.100/dab/docs//portaldab/documentos/atlas_hipertensao_diabetes.pdf.

Fernandes, B. S. M., Reis, I. A., \& Torres, H.C. (2016). Evaluation of the telephone intervention in the promotion of diabetes self-care: a randomized clinical trial. Rev. Latino-Am. Enfermagem [Internet], 24: e2719. http://www.revenf.bvs.br/scielo.php?script=sci_arttext\&pid=S0104-11692016000100396\&lng=pt.

Fragoso, L. V. C., Cunha, M. C. S. O., Fragoso, E. B., \& Araújo, M. F.M. (2019). Autocuidado em pessoas com diabetes mellitus tipo 1: vivências de adolescentes. Rev. pesqui. cuid. fundam. (Online), 289-296. http://www.seer.unirio.br/index.php/cuidadofundamental/article/view/6434/pdf_1.

Lim, A., \& Song, Y. (2020). The role of psychological insulin resistance in diabetes self-care management. Nurs Open., 7(3): 887-894. https://www.ncbi.nlm.nih.gov/pmc/articles/PMC7113521/.

Maia, M. A., Reis, I.A., \& Torres, H.C. (2016). Relationship between the users' contact time in educational programs on diabetes mellitus and self-care skills and knowledge. Rev. esc. enferm. USP [Internet], 50(1): 59-64. http://www.scielo.br/scielo.php?script=sci_arttext\&pid=S0080-62342016000100059\&lng=en.

Moreira, T. R., Toledo, L. V., Colodette, R. M., Mendonça, É. T. de, Amaro, M. de O. F., Ayres, L. F. A., \& Henriques, B. D. (2018). Fatores relacionados à autoaplicação de insulina em indivíduos com diabetes mellitus. Revista Gaúcha de Enfermagem, 39, e2017-0066. http://www.scielo.br/scielo.php?script=sci_arttext\&pid=S1983-14472018000100423\&lng=en\&nrm=iso.

Mukona, D., Munjanja, S. P., Zvinavashe, M., \& Stray-Pederson, B. (2017). Barriers of Adherence and Possible Solutions to Nonadherence to Antidiabetic Therapy in Women with Diabetes in Pregnancy: Patients' Perspective. Journal of Diabetes Research, vol. 2017. https://www.hindawi.com/journals/jdr/2017/3578075/.

Reis, P. dos, Arruda, G. O. de, Nass, E. M. A., Ratuchnei, E. S., Haddad, M. do C. F. L., \& Marcon, S. S. (2020). Autocuidado e percepção do tratamento para o diabetes por pessoas em uso de insulina. Rev. enferm. UFSM,10: 60. https://periodicos.ufsm.br/reufsm/article/view/39880/html.

Reis, P. dos, Marcon, S. S., Nass, E. M. A., Arruda, G. O. de, Back, I. R., Lino, I. G. T., Francisqueti, V. (2020). Desempenho de pessoas com diabetes mellitus na insulinoterapia. Cogitare enferm,25: e66006. em http://www.revenf.bvs.br/scielo.php?script=sci_arttext\&pid=S0103-21002020000100423.

Reis, P. dos, Marcon, S. S., Teston, E. F., Nass, E. M. A., Ruiz, A. G. B., Francisqueti, V., Silva, E. S., Otero, L. M. (2020). Intervenção educativa sobre o conhecimento e manejo de insulina no domicílio. Acta Paul. Enferm. (Online),33: eAPE20190241. http://www.revenf.bvs.br/scielo.php?script=sci_arttext\&pid=S0103-21002020000100450.

Rossaneis, M. A., Haddad, M. C. F. L., Mathias, T. A. de F., \& Marcon, S. S. (2016). Differences in foot self-care and lifestyle between men and women with diabetes mellitus. Revista Latino-Americana de Enfermagem, 24, e2761. https://www.scielo.br/scielo.php?script=sci_arttext\&pid=S0104-11692016000100384.

Santos, J. C. (2018). Comparação das estratégias educativas em diabetes mellitus: Educação em grupo e visita domiciliar. Universidade Federal de Minas Gerais. Belo Horizonte, s.n., 135. https://repositorio.ufmg.br/handle/1843/BUOS-B2ZMM3.

Souza, J. D., Baptista, M. H. B., Gomides, D. S., \& Pace, A. E. (2017). Adesão aos cuidados com o diabetes mellitus em três níveis de atenção à saúde. Esc. Anna Nery, 21 (4). https://www.scielo.br/scielo.php?script=sci_arttext\&pid=S1414-81452017000400232\&lng=en\&nrm=iso\&tlng=pt.

Teston, E. F., Peternella, F. M. N., Sales, C. A., Haddad, M. C. L., Cubas, M. R., \& Marcon, S. S. (2018). Efeito da consulta de enfermagem no conhecimento, qualidade de vida, atitude frente à doença e autocuidado em pessoas com diabetes. REME rev. min. enferm,22: e-1106. http://www.reme.org.br/artigo/detalhes/1242. 
Research, Society and Development, v. 10, n. 7, e24410716485, 2021

(CC BY 4.0) | ISSN 2525-3409 | DOI: http://dx.doi.org/10.33448/rsd-v10i7.16485

Vianna, M. S. (2016). Competência para o autocuidado na administração de insulina por septuagenários ou mais idosos. Universidade Federal de Minas Gerais. Belo Horizonte. https://repositorio.ufmg.br/bitstream/1843/ANDO-AAJHWT/1/mayara_sousa_vianna.pdf,

Vieira, G. de L. C., Cecílio, S. G., Torres, H. de C. (2017). A percepção dos usuários com diabetes sobre a estratégia de educação em grupos na promoção do autocuidado. Esc. Anna Nery Rev. Enferm,21(1): e20170017. https://www.scielo.br/scielo.php?script=sci_arttext\&pid=S1414-81452017000100217

Wu, S., Ell, K., Jin, H., Vidyanti, I., Chou, C.-P., Lee, P.-J., Gross-Schulman, S., Sklaroff, L. M., Belson, D., Nezu, A. M., Hay, J., Wang, C.-J., Scheib, G., Capua, P. D., Hawkins, C., Liu, P., Ramirez, M., Wu, B. W., Richman, M., Myers, C., Agustines, D., Dasher, R., Kopelowicz, A., Allevato, J., Roybal, M., Ipp, E., Haider, U., Graham, S., Mahabadi, V., \& and Guterman, J. (2018). Comparative Effectiveness of a Technology-Facilitated Depression Care Management Model in Safety-Net Primary Care Patients With Type 2 Diabetes: 6-Month Outcomes of a Large Clinical Trial. Journal of Medical Internet Research, 20 (4), e147. https://www.ncbi.nlm.nih.gov/pmc/articles/PMC5938593/. 